\title{
Lernen von der Natur - Biologie trifft Technik
}

S-Bahnhof Wildau. 40 Minuten von der Berliner City entfernt. Rote Backstein-Gebäude säumen den Weg. Große Hallen mit Glas und Stahl verbunden. Viele Grünflächen. Hier und da erkennt man noch Fragmente der historischen Lokomotiven-Herstellung. Tradition trifft auf Moderne. Hier mitten im Grünen und vor den Toren Berlins ist das Schülerlabor „Biologie trifft Technik“ an der Technischen Hochschule Wildau zuhause.

Durch die Initiative des Studiengangs Biosystemtechnik/Bioinformatik entstand 2008 das Schülerlabor „Biologie trifft Technik“. Zunächst als reine Vortragsreihe konzipiert, erweiterte sich das Angebot auf Nachfrage vieler SchülerInnen und LehrerInnen auch auf experimentelle Labormodule. Die Biosystemtechnik selbst ist ein relativ junges Wissenschaftsgebiet der Biotechnologie. Ihr Ziel ist es, biologische Systeme wie ganze Zellen, aber auch nur Zellbestandteile oder einzelne Moleküle mit technischen Bauelementen wie Siliziumchips zu kombinieren, d.h. Biologie trifft Technik. Solche biohybriden Systeme nutzen die hohe Spezifität und Sensitivität biologischer Systeme, um neue analytische Nachweisverfahren zu ermöglichen. So entstehen z.B. „elektrische Nasen“, die Rauschgift aufspüren und hochsensible „künstliche Zungen“, die Diabetes nachweisen können.

Um das weitgefächerte Themengebiet der Biosystemtechnik und ihrer angrenzenden Wissenschaften mehr publik zu machen und die SchülerInnen von heute als Studierende von morgen zu gewinnen, wurde durch Prof. Dr. Fred Lisdat (Professor Biosystemtechnik) das Schülerlabor „Biologie trifft Technik“ gegründet. Seit 2008 soll aber nicht nur selbstständig experimentiert, sondern insbesondere das Interesse an naturwissenschaftlich-technischen Themen in einem authentischen Rahmen vermittelt werden. Auch wenn der Name des Labors viele biologische Sachverhalte erahnen lässt, geht es in der Biologie nicht ohne Chemie, Physik, Informatik, Technik oder Mathematik. Interdisziplinarität bedeutet jedoch mehr als nur Biologie und Chemie miteinander zu verknüpfen. Vielmehr bietet es die Möglichkeit, bereits heute an kombinierten Lösungen im Grenzbereich zwischen Naturwissenschaft und Technik für künftige Problemstellungen zu forschen. Und so experimentieren die SchülerInnen in $2 \mathrm{er}$ Teams u.a. zu folgenden Themen: Wie funkti-

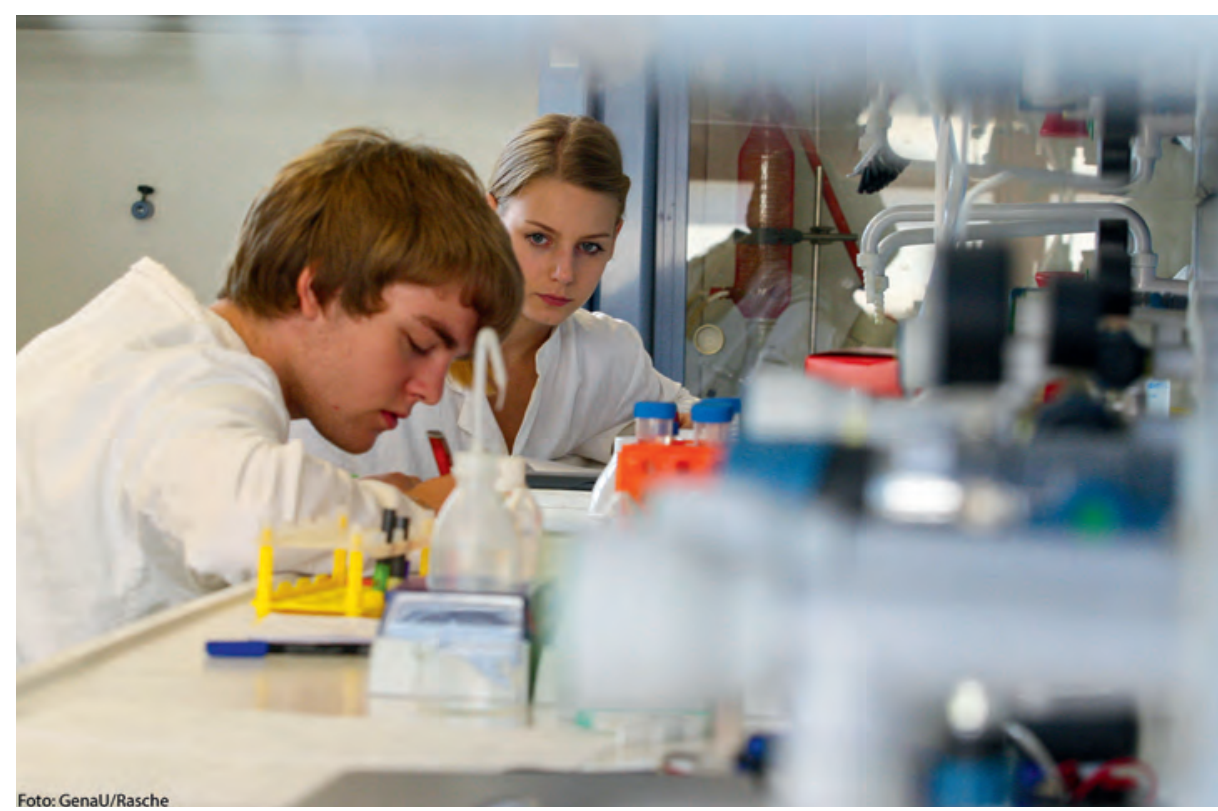

Auf die richtige Reihenfolge kommt es an: Vorbereitung - Experimentieren - Protokoll schreiben

Quelle: GenaU/Rasche

oniert ein kommerzieller „Blutzuckersensor“? Woher bekommt der Herzschrittmacher von morgen seine Energie? Kann man die Lichtenergie für einen Wecker nutzen? Womit lässt sich die Oberflächenstruktur von Zellen untersuchen?

Als klassisches Schülerlabor steht „Biologie trifft Technik“ SchülerInnen ab Klasse 10 zur Verfügung. Die verschiedenen Schnittstellen zu MINT-Fächern ermöglichen eine praktische Ergänzung zum theoretischen Schulunterricht. Alle praktischen Module verknüpfen mindestens drei Naturwissenschaften mit technischen Komponenten. Doch bevor es ans Experimentieren geht, kommen die MitarbeiterInnen von „Biologie trifft Technik“ in die Schule. Dort stellen sie die Hochschule, den Studiengang, sowie aktuelle Themen aus der eigenen Biosystemtechnik-Forschung vor. Es hat sich gezeigt, dass beim anschließenden Besuch im Schülerlabor die SchülerInnen lockerer und aufmerksamer beim Experimentieren sind, da sie das Team bereits kennen und über die Hintergründe zum Versuch bereits informiert sind. „Wir wollen zeigen, dass Wissenschaft und Forschung Spaß machen kann, und wir als Wissenschaftler auch von jungen Menschen wichtige Impulse für unsere Arbeit erhalten können. Kreative und vielleicht manchmal unkonventionelle Ideen haben schon immer zu Neuentdeckungen geführt.“, so Prof. Dr. Lisdat, Begründer des Schülerlabors.
Insgesamt erleben die SchülerInnen nicht nur Wissenschaft live, sondern erweitern auch ihre MINT-Kompetenzen. Gleichzeitig nutzen sie die Möglichkeit zur Studien- und Berufsinformation und um mit Studierenden und Wissenschaftlern ins Gespräch zu kommen. Denn nur wer von Naturwissenschaft und Technik begeistert ist, kann andere mit dieser Neugier anstecken. Bleiben Sie neugierig!

Anke Renger

\section{Kontakt}

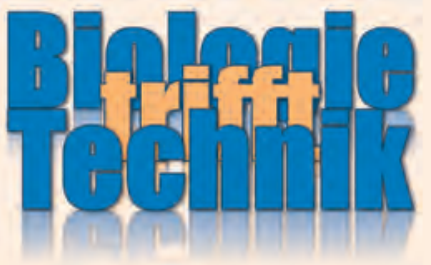

„Biologie trifft Technik“, Technische Hochschule Wildau, AG Biosystemtechnik Hochschulring 1

15745 Wildau

Tel.: 03375-508317

E-Mail: schuelerlabor@th-wildau.de www.th-wildau.de/bio-trifft-technik

Fachrichtungen: Biologie, Chemie, Physik, Technik, Informatik

Zielgruppen: ab Klasse 10 alle Schultypen; max. 18 TeilnehmerInnen 